\title{
Modelo de conocimiento y creatividad en la ingeniería de procesos. Un enfoque popperiano para desarrollo de productos de la mente humana
}

\section{RESUMEN}

La creatividad es un elemento primordial de la administración científica donde las decisiones se realizan mediante el paradigma racional que implica el empleo de criterios convergentes en la gestión, sistematización y jerarquía de problemas. A partirdel ingeniero industrial se han desarrollado diversas profesiones de ingeniería de procesos las cuales enfatizan el lado sistemático y racional, visible y concreto de su labor de ingeniero. La parte no visible lo constituye el lado heurístico y creador, que comprende la capacidad imaginativa y la visión divergente, que se desarrolla cuando surgen tareas nuevas y en áreas de investigación y diseño, donde se requiere visión e innovación. Este artículo intenta resaltar la capacidad creativa de una población de ingenieros industriales estudiando sus productos creativos, para detectar maneras de ver la realidad y su orientación creativa para la comprensión y resolución de problemas más allá de los enfoques tradicionales. Se observa que la creatividad en los ingenieros industriales deriva principalmente de su conocimiento verdadero, consecuente con la formación académica enfocada en gestionar la realidad para cambiarla y mejorarla, para beneficio de la sociedad.

Palabras clave: conocimiento verdadero, conocimiento falso, creatividad, producto intelectual

MODEL OF KNOWLEDGE AND CREATIVITY IN PROCESS ENGINEERING. A POPPERIAN APPROACH TO DEVELOPMENT OF HUMAN MIND PRODUCTS

\section{ABSTRAC}

Creativity is an essential element of scientific management where decisions are made by rational paradigm that involves the use of convergent criteria in the management, organization and hierarchy of problems. The process engineer emphasizes the systematic and rational side, which is the visible and concrete engineer's work. The invisible part constitutes the heuristic and creative side, comprising the imaginative and divergent view that complements the first and develops as new tasks arise in the areas of research and design, which requires vision and innovation. This paper aims to highlight the creativity of engineers. They have taken their creative products whose description and study, must lead to identifying new ways of seeing reality, beyond traditional approaches, and understand the orientation of creativity, to improve understanding and problem solving. Creativity in industrial engineers is based largely on True knowledge of reality which seems consistent with academic formation focused on managing reality to change and improve it, for the benefit of society.

Keywords: creativity, intellectual product, true knowledge, untrue knowledge

\section{INTRODUCCIÓN}

El presente estudio se refiere a la creatividad como elemento clave en el rol de gestión del ingeniero de procesos principalmente del ingeniero industrial, que le permite integrar la labor de diseño de productos, procesos, capacidad, layout y sistemas de control con la puesta en marcha y la operación de los sistemas productivos [5]. Estos sistemas pueden ser el puesto de trabajo, líneas de producción, empresas manufactureras o servicios, sectores o clúster industriales, sistemas sociales, sistemas conceptuales o cualquier tarea en cualquier nivel de resolución donde se desarrolle un modelo de causalidad, ya que estos abarcan todos los ámbitos de la actividad humana con fines productivos.

El objetivo general es mostrar que la creatividad es inherente a la labor del ingeniero industrial y la visualización de su importancia y de sus efectos, representando un renovado aporte para el conocimiento de la tarea de gestionar organizaciones.

Siendo la creatividad un importante elemento de la tarea de diseño se debe promover su conocimiento, desarrollo y aplicación mediante la difusión de los procesos creativos hacia la comunidad académica [4], lo que permitiría que se plasme una vertiente no explotada del ingeniero industrial: la imaginación y el conocimiento no sistemático al servicio del desarrollo de las organizaciones.

\section{PLANTEAMIENTO DEL ESTUDIO}

\subsection{Antecedentes}

Desde la aparición de la administración científica con Taylor, se ha enfatizado la aplicación del conocimiento científico para optimizar la productividad y eficiencia empresarial. El modelo racional ordenado, sistemático y reduccionista ha permitido el avance de la ciencia de la administración en diferentes direcciones. A partir de los experimentos Hawthorne se descubrieron las variables motivacionales e higiénicas del trabajo, con los estudios de las minas de Newcastle se diseñó el modelo socio técnico que integra las dimensiones técnica y social de la tarea productiva [9]. Su evolución exitosa ha consolidado las técnicas precisas

\footnotetext{
Doctor en Ingeniería, magíster en Administración, ingeniero industrial, economista. Profesor en la Facultad de Ingeniería Industrial-UNMSM. E-mail: aacevedo@speedy.com.pe, aacevedo@ industrial.unmsm.pe

** Magíster en Dirección de Empresas, ingeniero industrial, abogada. Profesora en UPG Facultad de Ingeniería de Sistemas e Informática UNMSM. E-mail: klinares@speedy.com.pe
} 
y racionales de la ciencia, pero ha dejado de lado o no ha permitido el crecimiento de técnicas heurísticas o intuitivas que son englobadas bajo la denominación general de creatividad.

Dentro de los antecedentes filosóficos se considera el planteamiento de Popper respecto a la relatividad del conocimiento cuando complementa y diferencia conocimiento e ignorancia, donde la sabiduría verdadera implica ser consciente de lo que se ignora por encima de lo que se sabe, poniendo como ejemplo a Sócrates cuando el oráculo Delphos lo elige como el hombre más sabio de Grecia [18]. Antes se diferencia el conocimiento verdadero del conocimiento falso o la vía de la verdad o episteme y la vía de la opinión o doxa [17]. Platón considera que la doxa comprende eikasia o imaginación y pistis o fe y creencias, aunque todo lo que la persona conoce y aplica, es considerado una creencia.

Otros antecedentes de creatividad en gestión, se encuentra el estudio de Acuña sobre las contribuciones de la literatura para el estudio de las organizaciones, donde plantea el empleo de la literatura para la formación de profesionales en administración, ya que las narraciones ofrecen un rico y valioso material para el aprendizaje de la vida laboral y para adquirir experiencia en gestión de organizaciones [2]. La Universidad de Chile ha publicado una antología sobre narraciones del trabajo, que recopila una serie de cuentos y brinda un retrato excepcional de los escenarios, eventos y personajes del entorno productivo, en conjunto presentan una perspectiva de la evolución empresarial y de los cambios sociales en la sociedad chilena del siglo veinte [2]. La obra de David Noonan contiene la perspectiva de diversos empresarios, académicos e investigadores sobre la influencia de las fábulas en los logros empresariales [15].

En el Perú, no existen antecedentes recientes sobre trabajos creativos que presenten un aspecto de la realidad empresarial. Se puede afirmar que la única obra referida a situaciones diversas, incluyendo las relacionadas a formas de hacer empresa y prácticas de negocio, se encuentra en la obra fundamental de Ricardo Palma, las Tradiciones Peruanas presentan conductas comúnmente aceptadas en el país, desde el siglo XVII hasta la fecha [16]. Pocos autores han tomado el tema de relaciones productivas y sociales, como Ciro Alegría que describe el mundo rural peruano frente a la urbe y los grupos dominantes, en el contexto del capitalismo de enclave en avance frente a las formas comunales prehispánicas en declive [3]. Existen narraciones menores que tocan el tema de las relaciones productivas nativas, marginales y desconocidos en el ambiente de gestión organizacional.

\subsection{El problema de investigación y objetivo}

La creatividad es un elemento primordial de la administración científica ya que el diseño de procesos y su implantación se realizan mediante el paradigma [12] racional que implica el empleo de criterios convergentes en la gestión, sistematización y jerarquía de problemas. Este foco en método y orden ha retrasado el desarrollo del paradigma heurístico e impedido el empleo de criterios divergentes en la labor creativa del ingeniero industrial. El rol del ingeniero industrial, plasmada en las visiones y misiones de los diversos centros académicos, enfatiza el lado sistemático y racional de la profesión, que se constituye en la parte visible y concreta de la labor del ingeniero. La otra parte no visible y diluida lo constituye el lado heurístico y creador, que comprende la capacidad imaginativa y la visión divergente, que complementa al primero y se desarrolla cuando el ingeniero se desenvuelve en tareas nuevas, en sectores emergentes, en investigación y diseño, dentro de actividades creativas e innovadoras.

La pregunta de investigación del estudio es: ¿De qué manera es el aporte del ingeniero industrial con enfoques divergentes y creativos en el conocimiento de la función empresarial? El problema secundario es: ¿Cómo es el aporte creativo de los ingenieros industriales?

El objetivo general es presentar antecedentes sobre el aporte creativo de ingenieros industriales sobre una situación real o ficticia, factible o no factible, teórica o empírica, sin rstricciones y bajo el único de criterio de empleo de la imaginación, independientemente del enfoque y la formación analítica de la profesión [13]. El objetivo específico es identificar narraciones, cuentos, u otros aportes sobre temas diversos y que no formen parte de la actividad central del ingeniero industrial.

\subsection{Importancia de la investigación}

El presente estudio se orienta a resaltar la capacidad creativa de los ingenieros industriales, con perspectiva y propósitos diversos y en contextos diferentes a los que se desenvuelve el ingeniero. Se han tomado los productos creativos aportados por ingenieros industriales, organizados bajos formas narrativas, cuyo descripción y estudio, ha de llevar a detectar nuevas maneras de ver la realidad, mas allá de los problemas tradicionales, y entender la orientación de la creatividad, para mejorar la comprensión y la resolución de problemas. 
Busca plantear los elementos de la creatividad que emplean los ingenieros industriales con diferentes propósitos y que les permite crear productos intelectuales que derivan de criterios divergentes y manifiestan cierto grado de innovación y aplicabilidad para la gestión empresarial [7], los que, de sistematizarse significarían un sustantivo aporte para ampliar las fronteras de conocimiento empírico, para la inclusión de enfoques divergentes en el estudio de los problemas, y para un cambio de perspectiva de los ingenieros industriales, cuando perciben, analizan, entienden y resuelven, creativamente, diversas situaciones problemáticas.

Constituye una investigación exploratoria para mostrar criterios inéditos [11] que conllevan nuevas y diversas perspectivas y modelos nuevos para la gestión de decisiones, los que se desarrollan y aplican por los ingenieros industriales. El diseño de nuevas metodologías basados en criterios heurísticos, mas allá de la racionalidad sistemática de la administración científica, implicaría una renovación en las técnicas y modelos de análisis y resolución de problemas, cambio en la percepción y entendimiento de las situaciones problemáticas.

\section{MARCO TÉORICO}

\subsection{Del Conocimiento y la Ignorancia}

Popper plantea que el conocimiento es relativo, donde lo importante no es la consciencia de lo que uno sabe sino la consciencia de la propia ignorancia. Complementa y diferencia conocimiento e ignorancia, donde la sabiduría verdadera implica ser consciente de lo que se ignora por encima de lo que se sabe, y pone el ejemplo de Sócrates cuando el oráculo Delphos lo elige como el hombre más sabio de Grecia [18]. Anteriormente, Parménides diferencia el conocimiento verdadero del conocimiento falso (vía de la verdad o episteme y vía de la opinión o doxa), Platón considera que la doxa comprende la eikasia o imaginación y la pistis o fe y creencias, aunque todo lo que la persona conoce y aplica, es considerado una creencia.

A partir del razonamiento que la ignorancia propia es infinita y cierta, y que el saber propio es limitado y refutable, plantea principios epistemológicos para la búsqueda de la verdad, los que pueden resumirse en:

- Principio de la falibilidad, donde uno o ambos, pueden tener la razón, o ambos estar equivocados.
- Principio del diálogo racional, poner a prueba las teorías mediante el razonamiento crítico y no mediante la crítica personal.

- Principio de acercamiento a la verdad, mediante las discusión impersonal y objetiva.

Estos principios implican centrar las discusiones en la razón (objetiva, por lo tanto demostrable) y no en los motivos (propósitos que son subjetivos y por lo tanto, relativos), además se confrontan conocimientos y no opiniones.

El conocimiento de las propias limitaciones se sustenta en que el conocimiento científico o cierto es solamente conjetural y se acepta como valedero mientras no sea refutado por otro conocimiento que supere las anomalías de las concepciones vigentes. Entonces el conocimiento total CT contiene elementos verdaderos $\mathrm{CV}$, elementos falsos CF y elementos no conocidos o ignorados $\mathrm{Cl}$, que se sintetiza en la fórmula:

$$
\mathrm{CT}=\mathrm{CV}+\mathrm{CF}+\mathrm{Cl}
$$

El conocimiento de la verdad implica tener consciencia de que el conocimiento puede ser verdadero cuando se sustenta en la episteme, hechos o fe, que corresponden a los paradigmas aceptados como verdaderos por ser demostrables (excepto la religión). El conocimiento es falso cuando se sustenta en la doxa, la seudociencia y la imaginación, que son no demostrables, comprende paradigmas subterráneos no aceptados (oficialmente). La Figura 1 muestra el modelo del conocimiento y la ignorancia.

Paradójicamente, el conocimiento no conocido o ignorado puede ser cognoscible o factible de conocerse en el futuro a partir del desarrollo de la ciencia o puede ser incognoscible e imposible de conocer por incapacidad de los sentidos para percibir fenómenos. Ver Tabla 1.

Mientras el conocimiento es limitado, la ignorancia es infinita, a medida que crece el conocimiento de las cosas, también crece el número de enigmas que se desconoce. La Figura 2 muestra gráficamente la manera en que el incremento de conocimiento conlleva nuevas preguntas no resueltas y nuevos problemas por resolver, lo que implica un incremento mayor de lo ignorado.

Todos los tipos de conocimiento CV, CF, Cl constituyen sistemas de paradigmas que permiten entender la realidad y resolver problemas, se emplean en las organizaciones para tomar decisiones y resolver situaciones problemáticas concretas. 
Figura 1. La relatividad del conocimiento y la ignorancia.
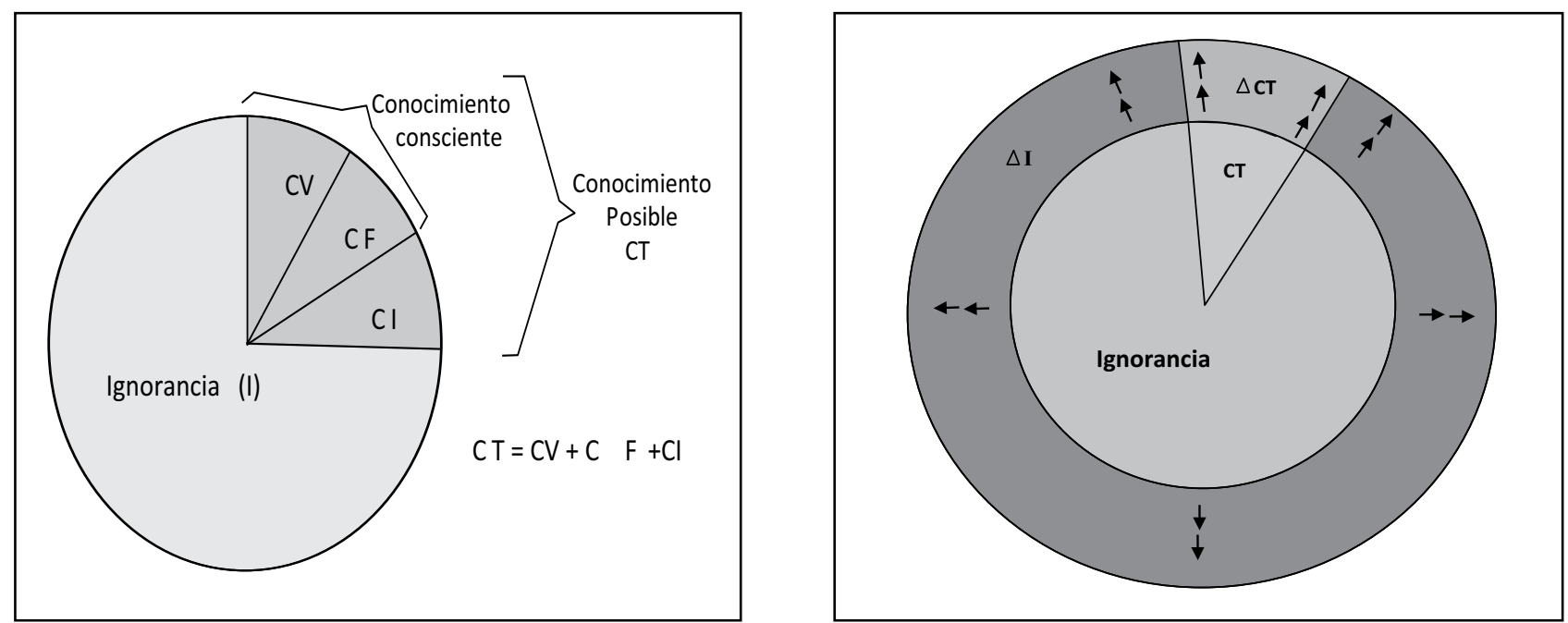

Tabla 1. Los tipos de conocimiento

\begin{tabular}{|c|c|c|c|}
\hline & Tipos & $\begin{array}{l}\text { Por su origen y } \\
\text { complejidad }\end{array}$ & Producto de la mente humana \\
\hline \multirow{5}{*}{$\begin{array}{l}\text { Conocimiento } \\
\text { total }\end{array}$} & \multirow{2}{*}{ Conocimiento verdadero } & Episteme & $\begin{array}{l}\text { Ciencia } \\
\text { Experiencia } \\
\text { Tecnología } \\
\text { Intuición }\end{array}$ \\
\hline & & $\mathrm{Fe}$ & $\begin{array}{l}\text { Religión } \\
\text { Filosofía } \\
\text { Derecho } \\
\text { Política }\end{array}$ \\
\hline & \multirow{2}{*}{ Conocimiento falso } & Doxa & $\begin{array}{l}\text { Opinión pública } \\
\text { Cultura de farándula } \\
\text { Cultura Internet, posmodernismo real } \\
\text { Instituciones-democracia }\end{array}$ \\
\hline & & Imaginación & $\begin{array}{l}\text { Arte } \\
\text { Folclore } \\
\text { Literatura } \\
\text { Futuro }\end{array}$ \\
\hline & Conocimiento ignorado & $\begin{array}{l}\text { Cognoscible } \\
\text { Incognoscible }\end{array}$ & $\begin{array}{l}\text { Factible de conocer en el futuro } \\
\text { Nunca se conocerá }\end{array}$ \\
\hline $\begin{array}{l}\text { Ignorancia } \\
\text { total }\end{array}$ & $\begin{array}{l}\text { Ignorancia verdadera } \\
\text { Ignorancia falsa } \\
\text { Ignorancia ignorada }\end{array}$ & & $\begin{array}{l}\text { Enigmas sin solución }(\mathrm{E}-\mathrm{T}) \\
\text { Enigmas resueltos } \\
\text { Infinito }\end{array}$ \\
\hline
\end{tabular}

Fuente: Elaboración propia. 


\subsection{El modelo del conocimiento}

Empleando un modelo gráfico elaborado a partir de los opuestos dialécticos de la función de la razón en el hacer ciencia (eje $\mathrm{x}$ ) donde los sueños, imaginación e invención y creación activa del conocimiento se enfrentan a la tabula rasa de la razón o percepción sensorial pasiva de la realidad, el eje y del mundo material donde las ideas y los conceptos de la existencia se oponen a a los fenómenos o manifestaciones de la realidad. Este modelo concuerda con el enfoque aristotélico de la verdad o del conocimiento verdadero [14]. Que se muestra en la Figura 3, dentro de las cuatro dimensiones epistemológicas de hacer-pensar y conocer-crear [1].

Con este esquema se definen los tipos de conocimiento falso de la Figura 4 , los cuales complementan al conocimiento verdadero. A la experiencia de la realidad se opone la percepción vulgar de la que derivan las creencias y simbolismos primitivos, a la episteme o ciencia demostrable se opone la doxa u opinión que no es conocimiento sino juicio subjetivo, y que al contrario de la ciencia no es descubrir la realidad sino manipularla. A la tecnología se opone la seudociencia como explicación de situaciones problemáticas perversas que no encuentran solución en el mundo real, $\mathrm{Al}$ conocimiento intuitivo, sistémico y relacional se le opone la imaginación que puede basarse en hechos realmente acontecidos o en ficción pura.

\subsection{Glosario}

Conocimiento falso. Doxa e imaginación incluyendo la seudociencia.

Conocimiento verdadero. Episteme y $\mathrm{Fe}$, incluyendo religión y filosofía.

Creatividad. Capacidad de producir ideas originales, trascendiendo métodos lógicos [10].

Doxa. Conocimiento derivado de la opinión vulgar.

Episteme. Conocimiento científico y estructurado .en el sentido aristotélico comprendería episteme, empeiria, tekne y noús.

Fe. Conocimiento verdadero, aceptado por sí mismo, que no requiere demostración.

Imaginación. Proceso mental abstracto que crea realidades nuevas o ficciones nuevas percibidas por la mente [6].

Intuición. Capacidad de conocer y decidir empleando procesos no sistemáticos ni racionalmente controlados o con información incompleta. Noús.
Noús. En su acepción aristotélica es el conocimiento sintético que permite realizar interrelaciones y crear soluciones creativas. En su acepción popperiana es el elemento conjetural por el cual se crea o se inventa el conocimiento.

Paradigmas. Modelos conceptuales estructurados como sistemas conceptuales estructurados que permiten percibir y entender la realidad para resolver problemas [12].

Productos de la mano humana. Bajo enfoque Engeliano, son productos tangibles derivados del trabajo [8]. Producto manual o manufactura.

Productos de la mente humana. Bajo enfoque Popperiano, son productos intelectuales del proceso de conocimiento. Ideas. [18]. Producto intelectual o mentefactura.

\section{EL MÉTODO DE INVESTIGACIÓN}

A partir de la hipótesis general: "La obra personal y creativa del ingeniero industrial tiene relevancia y representa un aporte sustantivo para el estudio de la gestión de las organizaciones", se elabora el presente estudio como investigación empírica empleando metodologías cualitativas para definir el modelo bajo el cual se desarrolla la creatividad y metodologías cuantitativas para corroborar la hipótesis bajo estudio.

En la investigación cualitativa se emplea el método hipotético-deductivo que permite percibir los hechos y formular las hipótesis preliminares planteando el modelo conceptual de creatividad con sus variables, luego se generan las hipótesis empíricas que serán corroboradas. Para la investigación cuantitativa se aplica el método inductivo-observacional, donde se obtienen y sistematizan los datos mediante el inventario de recolección de datos de los participantes del estudio, los que se emplean para la contrastación de las hipótesis, mediante herramientas de estadística inferencial.

Se emplea un diseño observacional tomando la población de productos mentales ofrecidos por los ingenieros industriales de la Facultad de Ingeniería Industrial de la UNMSM la cual es estudiada por una muestra de graduandos que determinan su finalidad y aporte frente a la gestión de organizaciones. La unidad de análisis es el producto intelectual (narraciones).

La población comprende a los ingenieros industriales que elaboran productos creativos que no tiene relación directa con la profesión. Estos productos de la mente humana o productos intelectuales se presentan como narraciones, cuentos, casos, 
Figura 3. El modelo del conocimiento verdadero aristotélico.

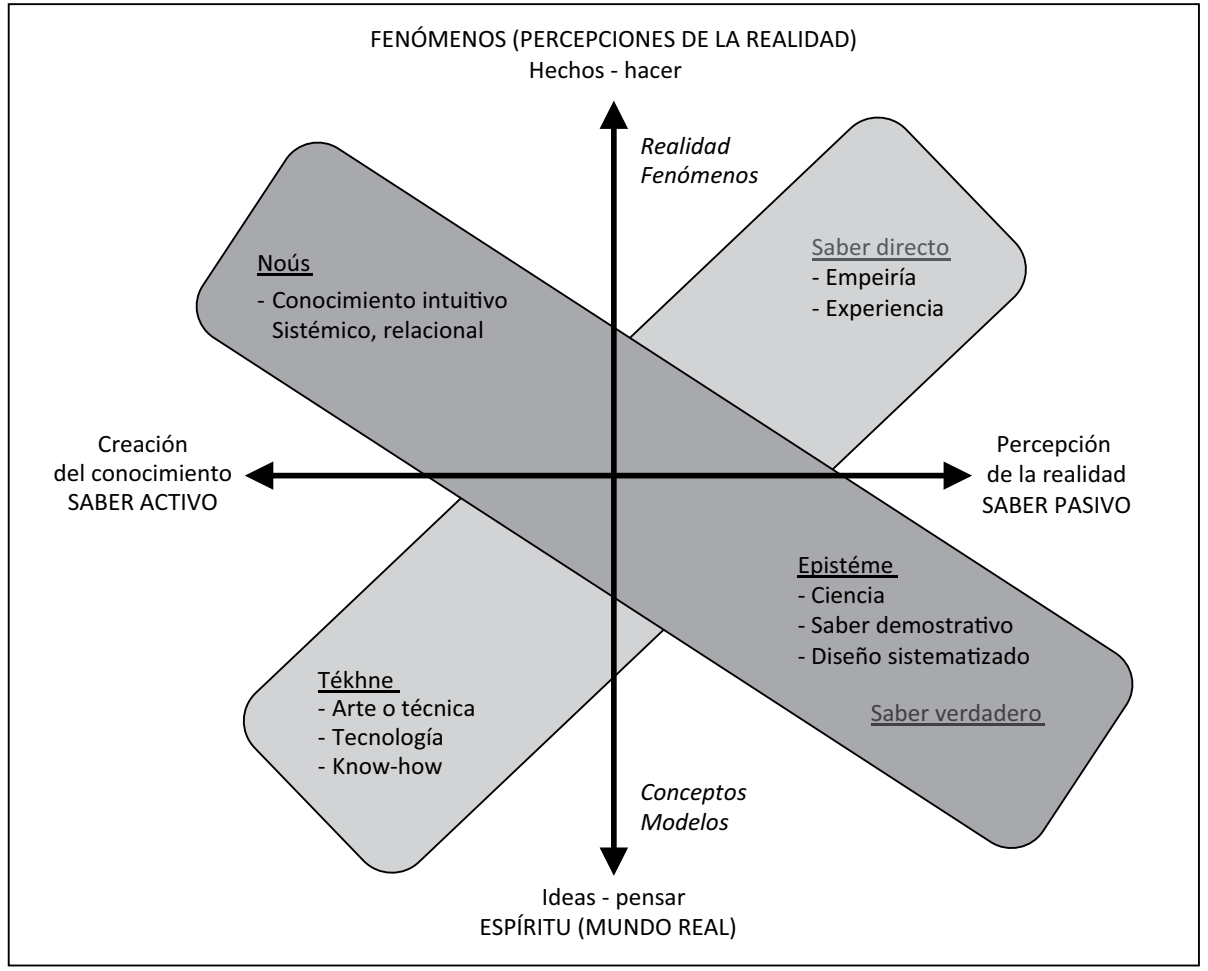

Fuente: Elaboración propia.

Figura 4. La Doxa o conocimiento falso.

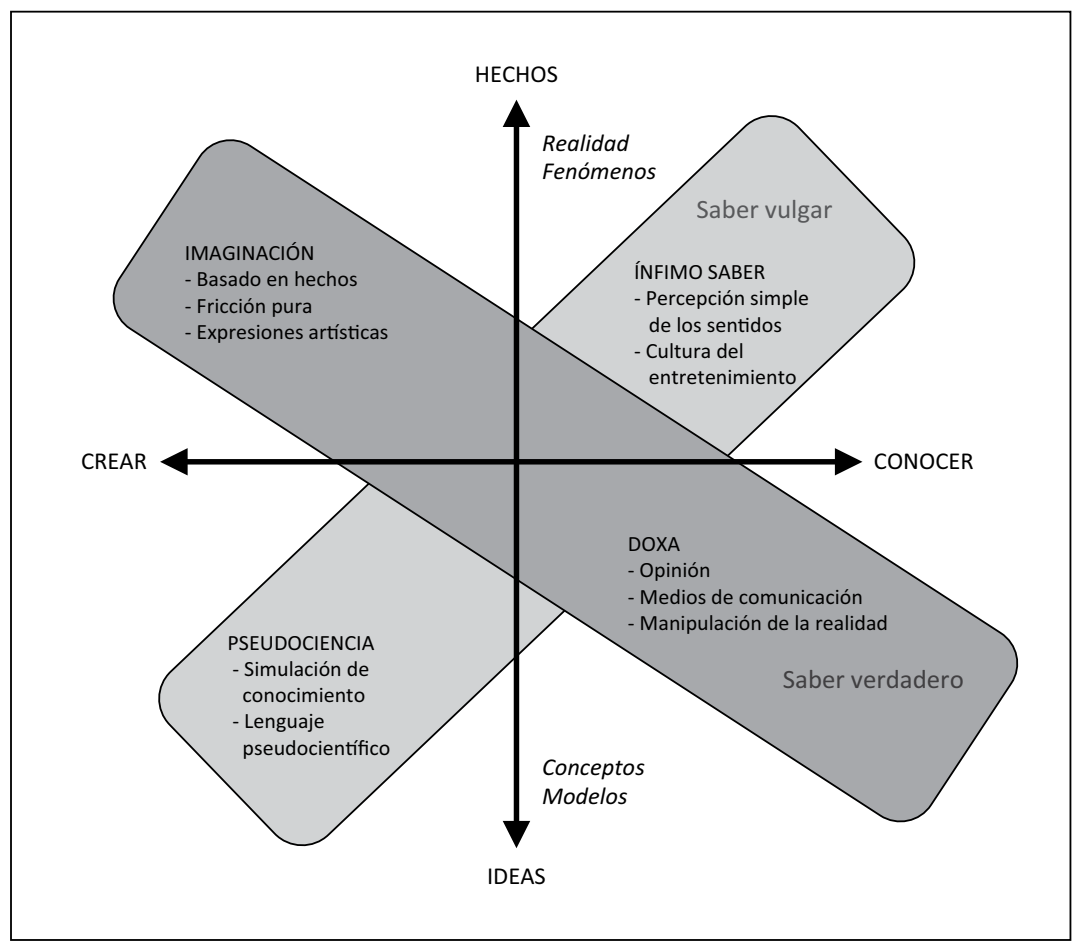

Fuente: Elaboración propia. 
poemas sobre cualquier tema. La recopilación de las narraciones se ha realizado por invitación abierta a todos los ingenieros industriales de la FII-UNMSM y la aplicación de la herramienta de recolección de datos, se realiza mediante un taller académico, aplicada sobre una muestra aleatoria.

El estudio de la finalidad y efecto en la gestión de organizaciones, de las narraciones de los ingenieros industriales, se realiza por un grupo definido de manera aleatoria, conformado por graduados universitarios, con diferente formación y experiencia y que no tienen relación con los ingenieros industriales de la población. El tamaño de muestra del grupo aleatorio de estudio denominado grupo-graduandos se ha determinado mediante estadística inferencial para pequeñas muestras donde no se conoce la desviación estándar con un nivel de confianza de $95 \%$.

\section{RESULTADOS DEL ESTUDIO}

Se emplea un indicador de creatividad construido como ratio producto intelectual sobre el total de docentes de la escuela en porcentaje. Es una medida de productividad de producto sobre recurso que va desde cero hasta 100 (máxima creatividad. De manera complementaria, es necesaria la identificación de la orientación de los ingenieros industriales en cuanto a la fuente de creatividad que emplean cuando desarrollan productos intelectuales y si esta orientación influye en la preparación y enseñanza de casos o situaciones empresariales problemáticas.

El planteamiento de la hipótesis se corrobora a partir del modelo de regresión lineal. La Hipótesis nula $\mathrm{H}_{0}$ es "La obra personal y creativa del ingeniero industrial no representa aporte sustantivo para el estudio de la gestión de las organizaciones", o el ingeniero industrial tiene preferencia irrelevante entre el conocimiento verdadero y conocimiento falso de manera que no se observa mayor aporte a la gestión de empresa. La Hipótesis alterna $H_{1}$ es "La obra personal y creativa del ingeniero industrial si representa un aporte significativo para el estudio de la gestión de las organizaciones" o la imaginación del ingeniero industrial se orienta a diferentes espacios, donde buscan un sentido pragmático cercano a la realidad, cuando ejercitan su capacidad de creatividad personal

La Hipótesis en el modelo se presenta:

$$
\begin{aligned}
& \mathrm{H}_{0}: \quad \mu_{1}=\mu_{2}=\mu_{3}=\mu_{4}=\mu \\
& \text { Todas las } \mu_{\mathrm{i}} \text { son iguales; donde } \mathrm{i}=1,2, \ldots \mathrm{k}, \mathrm{k} \\
& =4
\end{aligned}
$$

o: todas las medias de las $\mathrm{k}$ poblaciones son iguales

La Hipótesis alternativa es:

$$
\begin{aligned}
& H_{1}: \text { No todas las } \mu_{i} \text { son iguales } \\
& \text { o: } \exists \mathrm{i} / \mu_{\mathrm{i}} \neq \mu
\end{aligned}
$$

El resultado de la prueba $F$ para análisis de varianza de un factor se muestra en la Figura 5.

Para un nivel de significación (o probabilidad de error tipo I) de 0.05 el $\mathrm{F}$ calculado es superior al $\mathrm{F}$ teórico $(36.152>2.70)$.

- Se rechaza la hipótesis nula a un nivel de significación de 0.05 .

- Se acepta la hipótesis alternativa a un nivel de significación de 0.05 .

Por lo tanto, se rechaza la hipótesis nula que afirma que los ingenieros industriales no manifiestan preferencias por los diversos tipos de conocimiento y que no influyen en su percepción y definición de problemas. Alternativamente, se corrobora la hipótesis alterna, por lo tanto, el factor tipo de conocimiento tiene efecto significativo sobre la creatividad del ingeniero industrial,, ya que éste manifiesta preferencia por uno $u$ otro tipo de concimiento, lo influye en su percepción y definición de problemas

\section{DISCUSIÓN DE LOS RESULTADOS}

\subsection{Contenido empírico sobre creatividad en los ingenieros industriales}

En el grupo de ingenieros industriales donde existe creatividad, comprobable a través de sus productos intelectuales, se observa que tienen preferencia marcada hacia alguno de los diversos tipos de conocimiento. La Figura 6 muestra gráficamente la manera en que se corrobora la hipótesis alterna.

La creatividad se orienta a diferentes espacios. La Tabla 2 muestra la percepción del grupo acerca de los productos intelectuales o las narraciones elaboradas por los ingenieros industriales. Se considera que estos buscan un sentido pragmático y cercano a la realidad, cuando ejercitan su capacidad de creación e imaginación. La Figura 7, confirma la observación anterior, ya que el campo 2 referido al conocimiento epistémico contiene la mayor parte de narraciones.

Cabe destacar que las dimensiones que configuran las coordenadas del gráfico son el eje y o dimensión 
Figura 5. Prueba de hipótesis de la investigación -Creatividad de ii.

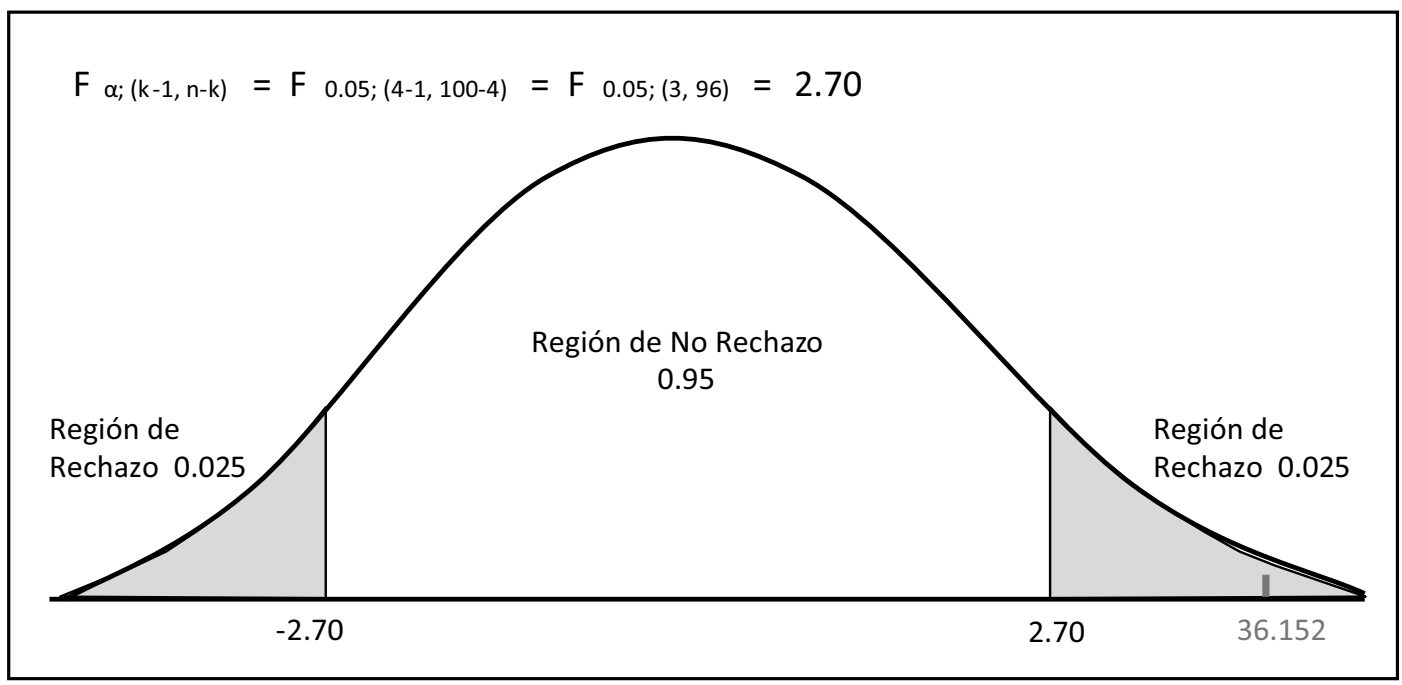

Fuente: Elaboración propia empleando SPSS.

Figura 6. Verificación gráfica de hipótesis.

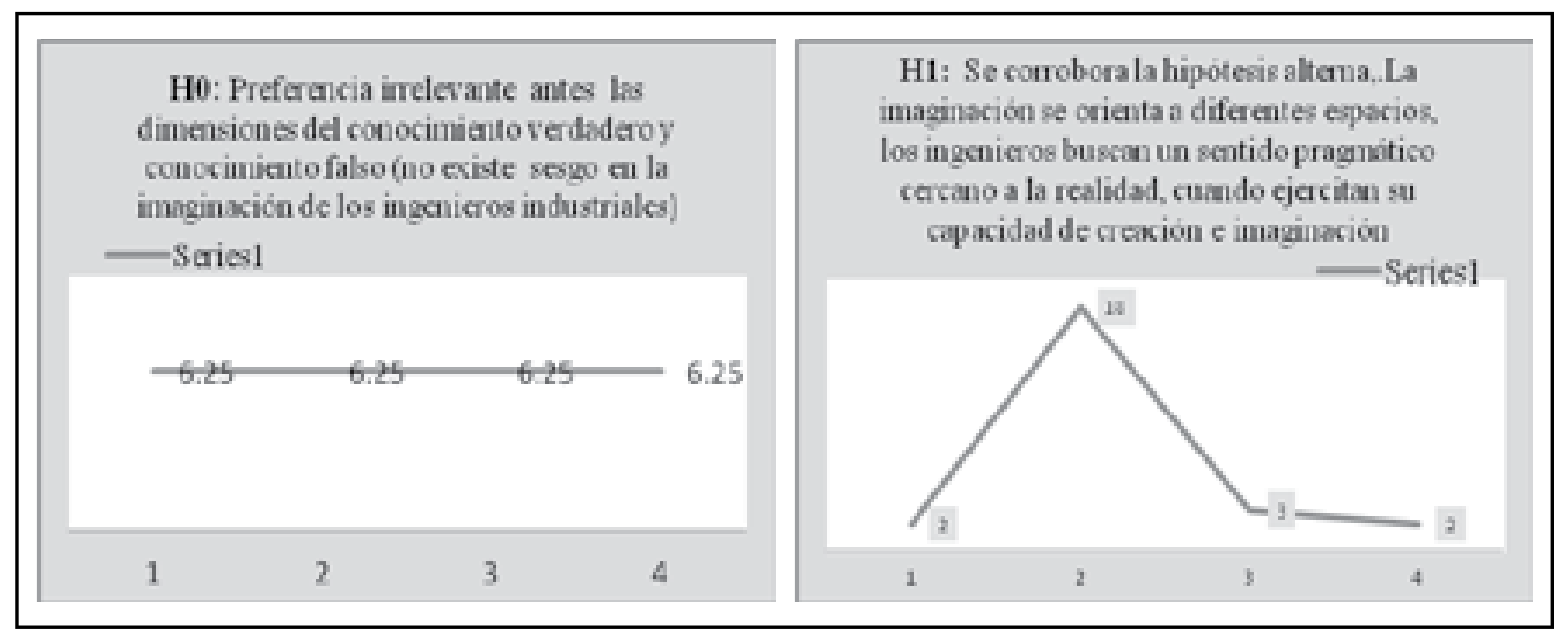

Fuente: Elaboración propia

del conocimiento (conocimiento verdaderoconocimiento falso) y el eje $\mathrm{x}$ o dimensión de la demostración (fenoménico-noúmeno) que permiten construir los cuatro campos de preferencia creativa del ingeniero industrial.

\subsection{Las fuentes de creatividad en el modelo de imaginación y creatividad}

En la Figura 8 se muestra el modelo de imaginación y creatividad referido a las raíces o fuentes de creatividad en el estudio. El $80 \%$ estima que los productos intelectuales se originan en el conocimiento verdadero de los hechos y de la ciencia, solamente el $20 \%$ considera que se basan en situaciones totalmente imaginarias.

Si se profundiza en el análisis, se añade mayor contenido empírico al modelo, ya que llega al detalles como el señalar que las narraciones muestran contextos demostrables de la realidad concreta, aparentemente no inventada sino descrita, lo que es consecuente con el enfoque pragmático enfocado en resultados que manifiestan los ingenieros industriales. 
Tabla 2. Producto intelectual-Creatividad de los ingenieros industriales

\begin{tabular}{|c|c|c|c|c|c|}
\hline & \multicolumn{4}{|c|}{ FUENTE DEL CONOCIMIENTO } & \multirow[b]{3}{*}{ Totales } \\
\hline & \multicolumn{2}{|c|}{ CO VERDADERO } & \multicolumn{2}{|c|}{ CO FALSO } & \\
\hline & FE & EPISTEME & $\begin{array}{l}\text { IMAGINA- } \\
\text { CIÓN }\end{array}$ & DOXA & \\
\hline \multicolumn{6}{|c|}{ PARTE I } \\
\hline 1. Profesor Emérito, P. Marín & 1 & 3 & & & 4 \\
\hline 2. La fiesta de la lluvia, R. Carrión & 1 & & 3 & & 4 \\
\hline 3. El hombre siniestro, A. Chung & 1 & 3 & & & 4 \\
\hline \multicolumn{6}{|c|}{ PARTE II } \\
\hline 4. La disyuntiva de Oswaldo, O. Cachay & & 2 & & 1 & 3 \\
\hline 5. El geniecillo valiente, C. Linares & & 4 & & 1 & 5 \\
\hline 6. Un buen empresario, A.Acevedo & & 4 & & & 4 \\
\hline \multicolumn{6}{|c|}{ PARTE III } \\
\hline 7. Fetos, A. Chung & 1 & 1 & 1 & & 3 \\
\hline 8. Sire Tobi: fiel amigo, P. Marín & 1 & 1 & 1 & & 3 \\
\hline $\begin{array}{l}\text { 9. La tumba con los tres muertos, } \mathrm{O} \text {. } \\
\text { Cachay }\end{array}$ & 2 & 1 & 2 & & 5 \\
\hline \multicolumn{6}{|c|}{ PARTE IV } \\
\hline 10. Cuando el tiempo nos alcance, P. Marín & & 5 & 1 & 1 & 7 \\
\hline 11. Espíritu empresarial, C. Linares & & 7 & & & 7 \\
\hline 12. Eternidad, A. Acevedo & 2 & 4 & & & 6 \\
\hline $\begin{array}{l}\text { 13. El libro de historia y el oro de la nación, } \\
\text { A. Acevedo }\end{array}$ & & 5 & & & 5 \\
\hline Totales por narración & 9 & 40 & 8 & 3 & 60 \\
\hline En \% & $15 \%$ & $67 \%$ & $13 \%$ & $5 \%$ & $100 \%$ \\
\hline Totales & \multicolumn{2}{|c|}{$49-82 \%$} & \multicolumn{2}{|c|}{$11-8 \%$} & $60-00 \%$ \\
\hline & \multicolumn{2}{|c|}{ CO VERDADERO } & \multicolumn{2}{|c|}{ CO FALSO } & \\
\hline
\end{tabular}

Fuente: Elaboración propia.

Los hallazgos de la investigación indican que existe un importante potencial creativo en el ingeniero industrial, el cual debe promoverse para desarrollar nuevos enfoques y métodos para la gestión, con criterios intuitivos y heurísticos que complementen las técnicas racionales que son parte de base de administración científica de la carrera de Ingeniería Industrial.

\section{REFERENCIAS BIBLIOGRÁFICAS}

[1] Acevedo, Adolfo (2013). La creatividad dentro de la labor del ingeniero industrial. Un enfoque divergente sobre el aprendizaje en gestión de organizaciones. Informe Técnico de Investigación. Instituto de Investigación de la Facultad de Ingeniería Industrial. Universidad Nacional Mayor de San Marcos UNMSM. Lima, Perú. 
Figura 7. Preferencia en Imaginación de ii

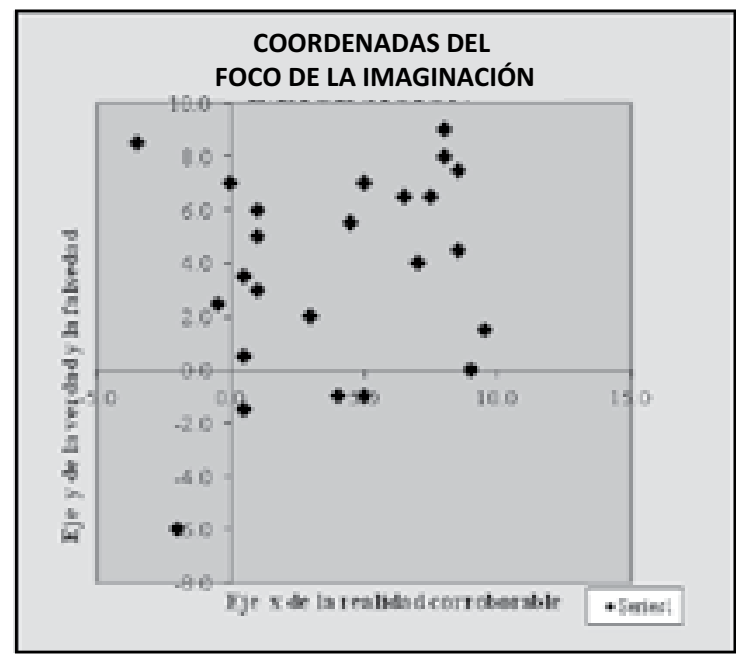

Fuente: Elaboración propia
Figura 8. Modelo de imaginación y creatividad

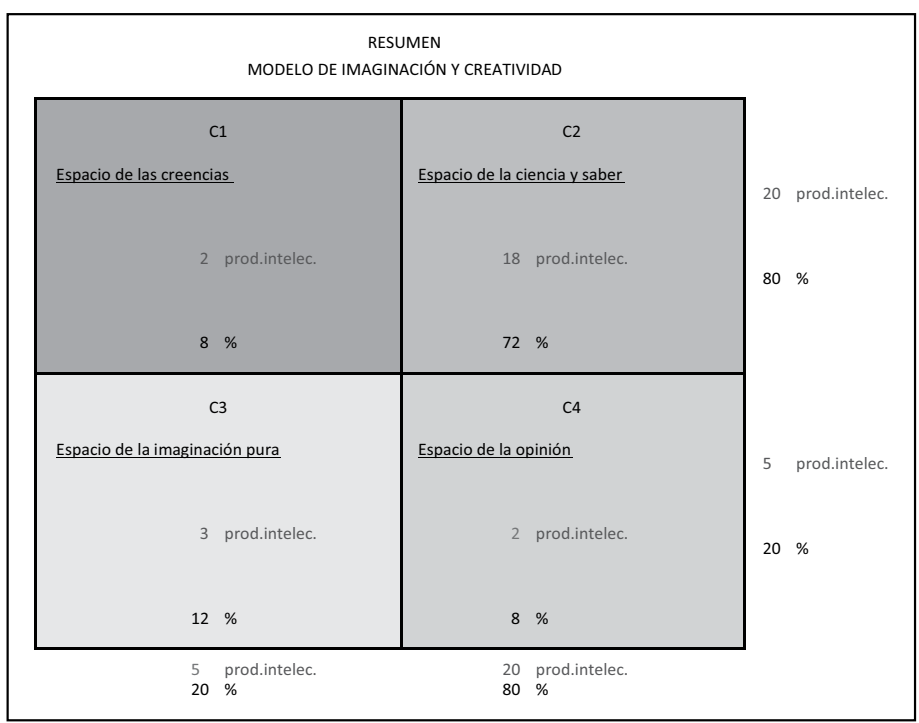

Fuente: Elaboración propia
[2] Acuña, Eduardo (2002). "Contribuciones de la literatura para el estudio de organizaciones". Estudios de Administración, Vol. 9, N. ${ }^{\circ}$ 2, 2002. Universidad de Chile, Facultad de Ciencias Económicas y Administrativas, Depto de Administración. Chile.

[3] Alegría, Ciro (2002). El mundo es ancho y ajeno. Ediciones PEISA. España.

[4] Arzola, Minerva (2010). Innovación, Competitividad y Desarrollo: Bases Conceptuales. Serie Cuadernos de Ingeniería Industrial $\mathrm{N}^{\circ}$ 9. Universidad el Carabobo. Venezuela.

[5] Bhidé, Amar (2000). Origen y evolución de nuevas empresas.Oxford University Press. México.

[6] De Bono, Edward (1970). El pensamiento lateral. Editorial Paidós, Barcelona.

[7] Drucker, Peter (2000). "La disciplina de la innovación" en HBR, Creatividad e innovación. Ediciones Deusto S.A. España.

[8] Engels (1955). El papel del trabajo en la transformación del mono en hombre. 1ra. Edición. Editorial Progreso. Moscú.

[9] Escorsa y Valls (2001). Tecnología e innovación en la empresa. Dirección y gestión. Alfaomega Grupo Editor S.A. de C.V. México D.F.

[10] HBR. Harvard Business Review (2000). Creatividad e innovación. Ediciones Deusto S.A. Bilbao, España.
[11] Hernández, Sampieri, Fernández, Baptista (2010). Metodología de la investigación. Quinta edición. Ed. Mcgraw Hill, Chile.

[12] Kuhn, Thomas (2006). La Estructura de las Revoluciones Científicas. 3ra. Edición. Fondo de Cultura Económica. México.

[13] Morgan, Gareth (1999). IMAGI-N-I-ZACIÓN. EDICIONES GRANICA S.A. Barcelona, España.

[14] Navarro y Calvo (1990). Historia de la Filosofía. Grupo Anaya S.A. España.

[15] Noonan, David (2005). Esopo, lecciones de negocio poderosas. Editorial Caribe, Inc. Nahville, Tenneessee, USA.

[16] Palma, Ricardo (1958). TRADICIONES PERUANAS. EDITORIAL CODEX S.A. Buenos Aires. Argentina.

[17] Parménides. Extraído el 15-08-2013 de http:// es.wikipedia.org/wiki/Parm\%C3\%A9nides_de_ Elea

[18] Popper, Karl (1991). EL CONOCIMIENTO DE LA IGNORANCIA. Diario 16 de Madrid. Extraído el 12-05-2013 de: http://www.unalmed.edu.co/ poboyca/ documentos/Doc.\%20Seminario\%20I/EL_ CONOCIMIENTO_DE_LA_IGNORANCIA. pdf. http://elpais.com/diario/1991/10/29/ cultura/688690809_850215.html. 